\title{
Spatial and temporal action of chicken primordial germ cells during initial migration
}

\author{
Kyung Soo Kang*, Hyung Chul Lee*, Hyun Jeong Kim, Hyo Gun Lee, Young Min Kim, Hong Jo Lee, \\ Young Hyun Park, Seo Yeong Yang, Deivendran Rengaraj ${ }^{1}$, Tae Sub Park ${ }^{2}$ and Jae Yong Han \\ Department of Agricultural Biotechnology, College of Agriculture and Life Sciences, and Research Institute of \\ Agriculture and Life Sciences, Seoul National University, Seoul 151-921, Korea, ${ }^{1}$ Department of Animal Science and \\ Technology, Chung-Ang University, Anseong 456-756, Korea and ${ }^{2}$ Graduate School of International Agricultural \\ Technology and Institute of Green-Bio Science and Technology, Seoul National University, Pyeongchang-gun, \\ Gangwon-do, Seoul 232-916, Korea
}

Correspondence should be addressed to J Y Han; Email: jaehan@snu.ac.kr

*(K S Kang and H C Lee contributed equally to this work)

\begin{abstract}
In most animals, primordial germ cells (PGCs) originate from an extragonadal region and migrate across the embryo to the gonads, where they differentiate and function. During their migration, PGCs move passively by morphogenetic movement of the embryo or move actively through signaling molecules. To uncover the underlying mechanism of first-phase PGC migration toward the germinal crescent in chickens, we investigated the spatial and temporal action of PGCs during primitive streak formation. Exogenously transplanted PGCs migrated toward the anterior region of the embryo and the embryonic gonads when they were transplanted into the subgerminal cavity, but not into the posterior marginal zone, in Eyal-Giladi and Kochav stage $\mathrm{X}$ embryos. These results indicate that for passive migration toward the anterior region the initial location of PGCs should be the central region. Notably, although PGCs and DF-1 cells migrated passively toward the anterior region, only PGCs migrated to the germinal crescent, where endogenous PGCs mainly reside, by active movement. In a live-imaging experiment with green fluorescence protein-expressing transgenic embryos, exogenous PGCs demonstrated markedly faster migration when they reached the anterior one-third of the embryo, while somatic cells showed epiblast movement with constant speed. Also, migrating PGCs exhibited successive contraction and expansion indicating their active migration. Our results suggest that chicken PGCs use sequential passive and active forces to migrate toward the germinal crescent.
\end{abstract}

Reproduction (2015) 149 179-187

\section{Introduction}

In embryogenesis, either passive or active migration of cells plays a central role for the development and morphogenesis of organisms. During passive migration, cells are transferred by motile neighboring cells. In contrast, the actively migrated cells move via a dynamic and integrated system including morphological polarization, membrane extension, and change in moving speed governed by various molecular mechanisms (Lauffenburger \& Horwitz 1996). In particular, spatially and temporally appropriate migration of cells in embryogenesis is closely related to their fate decision. Thus, studies on cell migration mechanism help us to understand the characteristics and differentiation of cells.

In most animals, primordial germ cells (PGCs), the precursors of functional gametes, are segregated from somatic cell lineages in the initial developmental phase, although they are specified by two different mechanisms in different species: preformation and induction (Extavour \& Akam 2003). In addition to their early segregation, one of the conserved and striking features of PGCs is their unique migration pathway. During migration toward their destination, PGCs are regulated by intrinsic and extrinsic guidance mechanisms, which utilize signaling pathway-related and adhesion-related molecules (Richardson \& Lehmann 2010). Because proper cell migration is crucial for normal embryo development and tissue differentiation (Franz et al. 2002), studying the migration mechanism of PGCs is also important for understanding the biology of other migrating cell types.

Chicken PGCs are normally located in the central region of the area pellucida, which contains both the epiblast and hypoblast before primitive streak formation (Ginsburg \& Eyal-Giladi 1987), and initiate their migration from this region. Chicken PGCs have three major migratory routes before they settle within the 
developing gonads. First, they migrate from the central region of the area pellucida at Eyal-Giladi and Kochav (EGK) stage $X$ to the germinal crescent at Hamburger and Hamilton (HH) stage 4 (Hamburger \& Hamilton 1951, Eyal-Giladi \& Kochav 1976, Ginsburg \& Eyal-Giladi 1986). Second, they undergo circulation in extraembryonic blood vessels during $\mathrm{HH}$ stages 12-15 (Swift 1914, Fujimoto et al. 1976). Finally, they settle down in the genital ridges at $\mathrm{HH}$ stage 17 (Fujimoto et al. 1976). Several studies have uncovered the mechanisms by which the PGCs become lodged in the vascular system and colonize the gonads (Stebler et al. 2004, De Melo Bernardo et al. 2012). However, how they migrate toward the anterior region called the germinal crescent is less well studied.

To elucidate the mechanism of chicken PGC migration, we conducted spatial and temporal analyses during their first-phase migration from the central region of the area pellucida toward the germinal crescent. We investigated the migration route of fluorescently labeled exogenous PGCs that were transplanted into EGK stage $X$ embryos and compared it with that of endogenous PGCs and somatic cells. Based on our results, we explain that passive and active forces sequentially regulate the migration of PGCs toward the germinal crescent.

\section{Materials and methods}

\section{Experimental animals and animal care}

White Leghorn chickens were managed according to our standard operation protocols, and all experimental procedures were approved by the Institutional Animal Care and Use Committee of Seoul National University, before the experiments were performed (SNU-070823-5). Transgenic (TG) embryos expressing green fluorescent protein (GFP) under the control of the cytomegalovirus (CMV) promoter were derived in our previous study (Park \& Han 2012).

\section{Whole-mount in situ hybridization}

To make hybridization probes, total RNA from magnetic-activated cell sorting-positive PGCs at E6.5 was reverse-transcribed, and the resulting cDNA was amplified using chicken deleted in azoospermia-like (cDAZL)-specific primers (F, 5'-CGT CAA CAA CCT GCC AAG GA-3' and R, 5'-TTC TTT GCT CCC CAG GAA CC-3'; product size 540 bp), as described previously (Rengaraj et al. 2010). PCR products of the correct size were cloned into the pGEM-T vector (Promega). After sequence verification, the recombinant plasmids containing the gene were amplified using T7- and SP6-specific primers (T7, 5'-TGT AAT ACG ACT CAC TAT AGG G-3' and SP6, 5'-CTA TTT AGG TGA CAC TAT AGA AT-3') to prepare the template for labeling the hybridization probes. Digoxigenin (DIG)-labeled sense and antisense $c D A Z L$ hybridization probes were transcribed in vitro using a DIG RNA labeling kit (Roche Diagnostics). A standard protocol for whole-mount in situ hybridization in chickens was followed (Stern 1998).

\section{Migration assay}

In vitro cultured PGCs with or without GFP expression (Park \& Han 2012) and DF-1 cells (a continuous chicken embryonic fibroblast cell line) were labeled with $\mathrm{PKH} 26$ fluorescent dye (Sigma-Aldrich) and then transferred into the subgerminal cavity or the posterior marginal zone (PMZ) of EGK stage $X$ embryos. After sealing with Parafilm, eggs were further incubated at 37$38{ }^{\circ} \mathrm{C}$ under $60-70 \%$ relative humidity until $\mathrm{HH}$ stage 4 (18 h of incubation) or 10 (34 h of incubation) (Hamburger \& Hamilton 1992). The embryos were detached from the yolk and transferred to saline to facilitate the removal of the yolk and vitelline membrane. To assay migration into the gonads, eggs were incubated for an additional 6 days until stage 28. Then, gonads and mesonephric kidneys were retrieved from the recipient embryos. The harvested embryos and gonads were fixed with $4 \%(\mathrm{w} / \mathrm{v})$ paraformaldehyde in $1 \times$ PBS for further immunostaining or observed under an AZ100 multipurpose zoom confocal microscope (Nikon Corporation, Tokyo, Japan).

\section{Whole-mount immunohistochemistry with $c D A Z L$ antibody}

The polyclonal antibodies against CDAZL were produced by immunizing host rabbits with a synthetic peptide corresponding to residues near the N-terminus of cDAZL (SANAEAQCGSISEDNTH, amino acids 2-17). The specificity of CDAZL antibody on chicken PGCs was tested by co-immunostaining of in vitro cultured PGCs with anti-SSEA1 and CDAZL antibodies (Santa Cruz Biotechnology; Supplementary Fig. S1, see section on supplementary data given at the end of this article). To label endogenous PGCs, the harvested whole-mount embryos were washed three times with PBS and blocked with blocking buffer (PBS containing 5\% goat serum and 1\% BSA) for $1 \mathrm{~h}$ at room temperature. The samples were then incubated at $4{ }^{\circ} \mathrm{C}$ overnight with rabbit anti-cDAZL. After washing with PBS three times, embryos were incubated with secondary antibodies labeled with FITC (Santa Cruz Biotechnology) for $4 \mathrm{~h}$ at room temperature. The embryos were washed with PBS three times and visualized under the multipurpose zoom confocal microscope.

\section{Time-lapse imaging with ex-ovo culture and migration assays}

PKH26-labeled PGCs were transplanted into the subgerminal cavity of GFP_TG embryos at EGK stage X and ex ovo cultured for time-lapse imaging using a confocal microscope. The ex ovo culture was performed using an improved shell-less culture system (Yalcin et al. 2010). Briefly, cling wrap was affixed circumferentially on plastic cups containing warm sterile water using a rubber band to make a hammock. The embryos with yolks and albumin were placed onto the hammock. Then, a Petri dish was placed on top of the cup to seal the embryo. The embryo with the best morphology was selected and cultured in a Chamlide incubator (Live Cell Instrument, Seoul, Korea) at $37.8^{\circ} \mathrm{C}$. Time-lapse images were taken using the AZ100 multipurpose zoom confocal microscope. To track selected cells, manual tracking was performed using NIS-elements (Nikon) according to the manufacturer's guidelines. The centers 


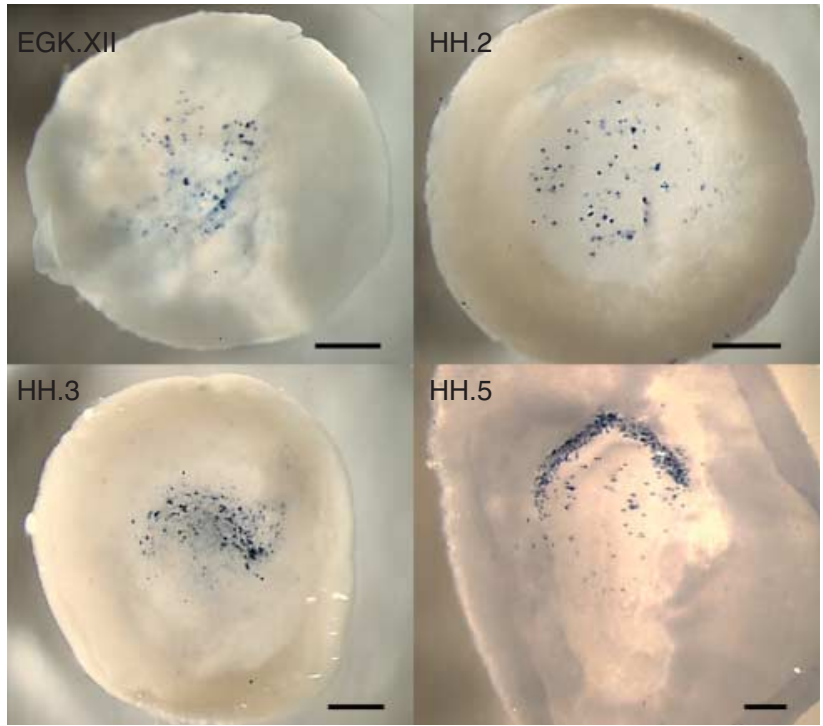

Figure 1 Distribution of PGCs during primitive streak formation. Whole embryos collected at EGK stage XII to $\mathrm{HH}$ stage 5 were hybridized with antisense probes for $C D A Z L$. At $\mathrm{HH}$ stages 2 and 3, PGCs migrating toward the anterior region were observed. At $\mathrm{HH}$ stage 5, PGCs gathered in the germinal crescent were detected. The anteroposterior axis describes the areas above and below the area of primitive streak formation. Scale bars $=500 \mu \mathrm{m}$.

of the manually selected cells were designated at each time point and the velocity of the cells was calculated based on the distance between their positions at two consecutive time points.

\section{Statistical analyses}

Statistical Analysis System software (SAS Institute, Cary, NC, USA) was used for analysis of all numerical data. Treatments were compared using the least-square difference or Duncan method, and ANOVA was used to determine the significance of the main effects. The level of significant differences was set at $P<0.05$.

\section{Results}

\section{Distribution of PGCs during primitive streak formation}

To identify PGCs in whole embryos, $c D A Z L$ probes were used as a PGC-specific marker. At EGK stage XII (2 h of incubation), most of the PGCs were located in the central region. At $\mathrm{HH}$ stage 2 (6 h of incubation), some of PGCs exhibited anterior position. At $\mathrm{HH}$ stage 3 (12 $\mathrm{h}$ of incubation), many PGCs were gathered in the anterior region. At HH stage 5 (22 h of incubation), most of the PGCs were clearly located in the germinal crescent region (Fig. 1).

\section{Passive migration of PGCs depends on their initial location}

To investigate the migratory pathway of PGCs during their first-phase of migration, PKH26-labeled exogenous PGCs were transplanted into the subgerminal cavities of EGK stage $X$ embryos (Fig. 2A). During incubation, exogenous PGCs migrated and became located in the marginal area of $\mathrm{HH}$ stage 4 embryos, mostly in the anterior region (Fig. 2B). When PKH26-labeled DF-1 cells were transplanted as a control, they migrated and became located in the extreme marginal area of the embryo at $\mathrm{HH}$ stage 4 (Fig. 2C). Thus, both exogenous PGCs and DF-1 cells migrated toward the anterior marginal zone when they were transplanted into the subgerminal cavities of EGK stage $X$ embryos $(n>4$ for both PGCs and DF-1 cells).
A

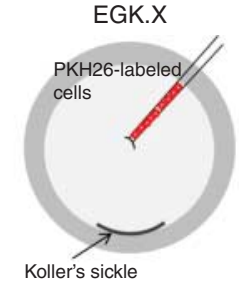

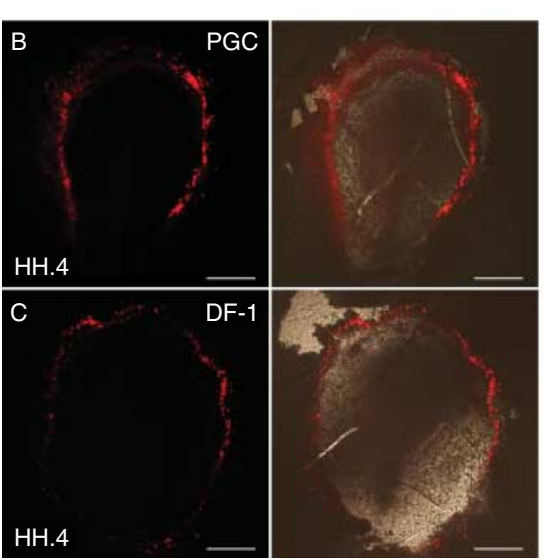

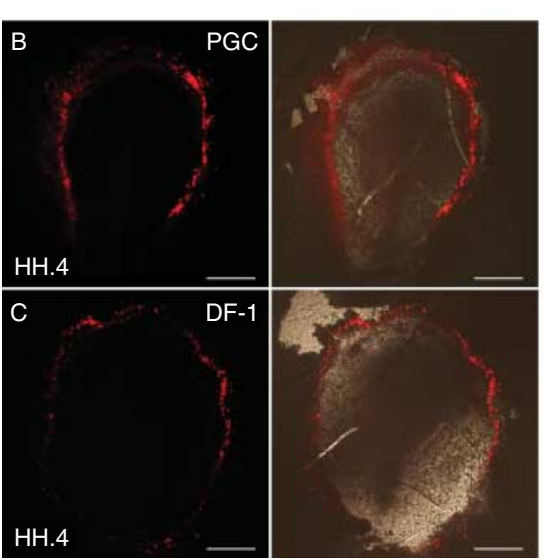

Anterior (A)

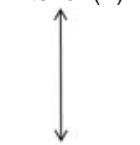

Posterior (P)
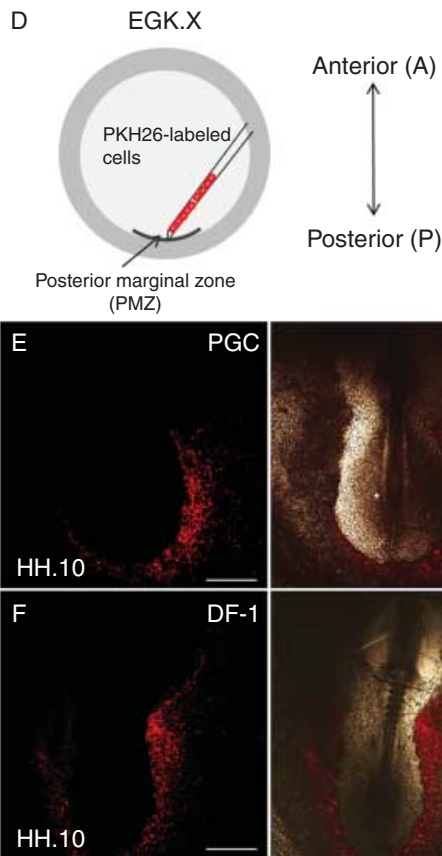

Posterior $(\mathrm{P})$

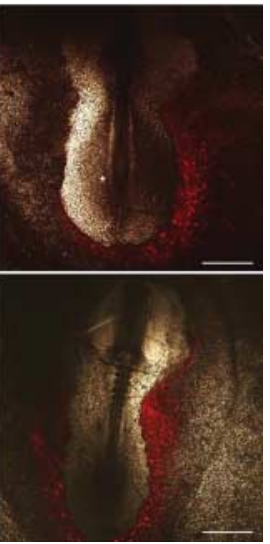

Figure 2 Effect of initial PGC location on passive migration toward the anterior marginal region. After PKH26 labeling, 1000 PGCs or DF-1 cells were transplanted into the subgerminal cavity $(\mathrm{A}, \mathrm{B}$, and $\mathrm{C})$ or the $\mathrm{PMZ}(\mathrm{D}, \mathrm{E}$, and $\mathrm{F})$ in EGK stage $X$ embryos. After incubation, the embryos were observed under a microscope at $\mathrm{HH}$ stage 4 or $\mathrm{HH}$ stage 10 . When transplanted into the subgerminal cavity, both PGCs (B) and DF-1 cells (C) migrated toward the anterior region of embryos at $\mathrm{HH}$ stage 4. When transplanted into the PMZ, both PGCs (E) and DF-1 cells $(F)$ reached the posterior half of the embryo. PMZ, posterior marginal zone; scale bars $=1000 \mu \mathrm{m}$. 


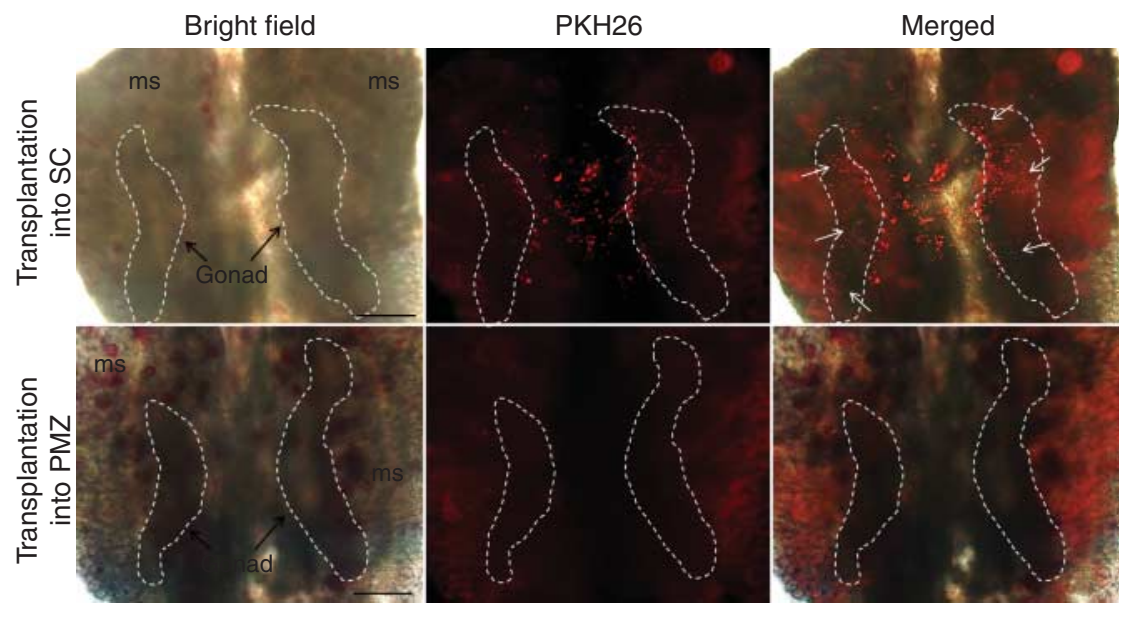

Figure 3 Effect of initial PGC location on migration toward the gonads. Approximately 5000 PGCs were labeled with PKH26 and transplanted into the subgerminal cavity (upper panels) or the PMZ (lower panels) in EGK stage $X$ embryos. After 6 days of incubation, the embryonic gonads and mesonephric kidneys (ms) were observed under a microscope. Migrating PGCs were only detected in the embryonic gonads (white arrows) when they were transplanted into the subgerminal cavity. The dotted lines indicate the embryonic gonads. PMZ, posterior marginal zone; scale bars $=500 \mu \mathrm{m}$.
Next, to access the ability of PGCs to migrate toward the anterior region, $\mathrm{PKH} 26$-labeled cells were transplanted into the posterior end (PMZ) of EGK stage $X$ embryos (Fig. 2D). The embryos were further incubated until $\mathrm{HH}$ stage 10 to give PGCs more time to migrate toward the anterior region $(n>4)$. At HH stage 10, PGCs had not reached the anterior region, but they had migrated and were located in the posterior half of the embryo (Fig. 2E). Exogenous DF-1 cells showed a similar pattern to PGCs ( $n>4$; Fig. $2 \mathrm{~F})$.
When the embryos were further incubated until the embryonic day 6, PGCs transplanted into the subgerminal cavity migrated toward the embryonic gonads $(n>20)$. In contrast, PGCs transplanted into the PMZ did not migrate toward the gonads (Fig. 3). The above experiments confirm that PGCs transplanted into the subgerminal cavity could only migrate passively toward the anterior region of the embryo during hypoblast formation and contribute to germline development.
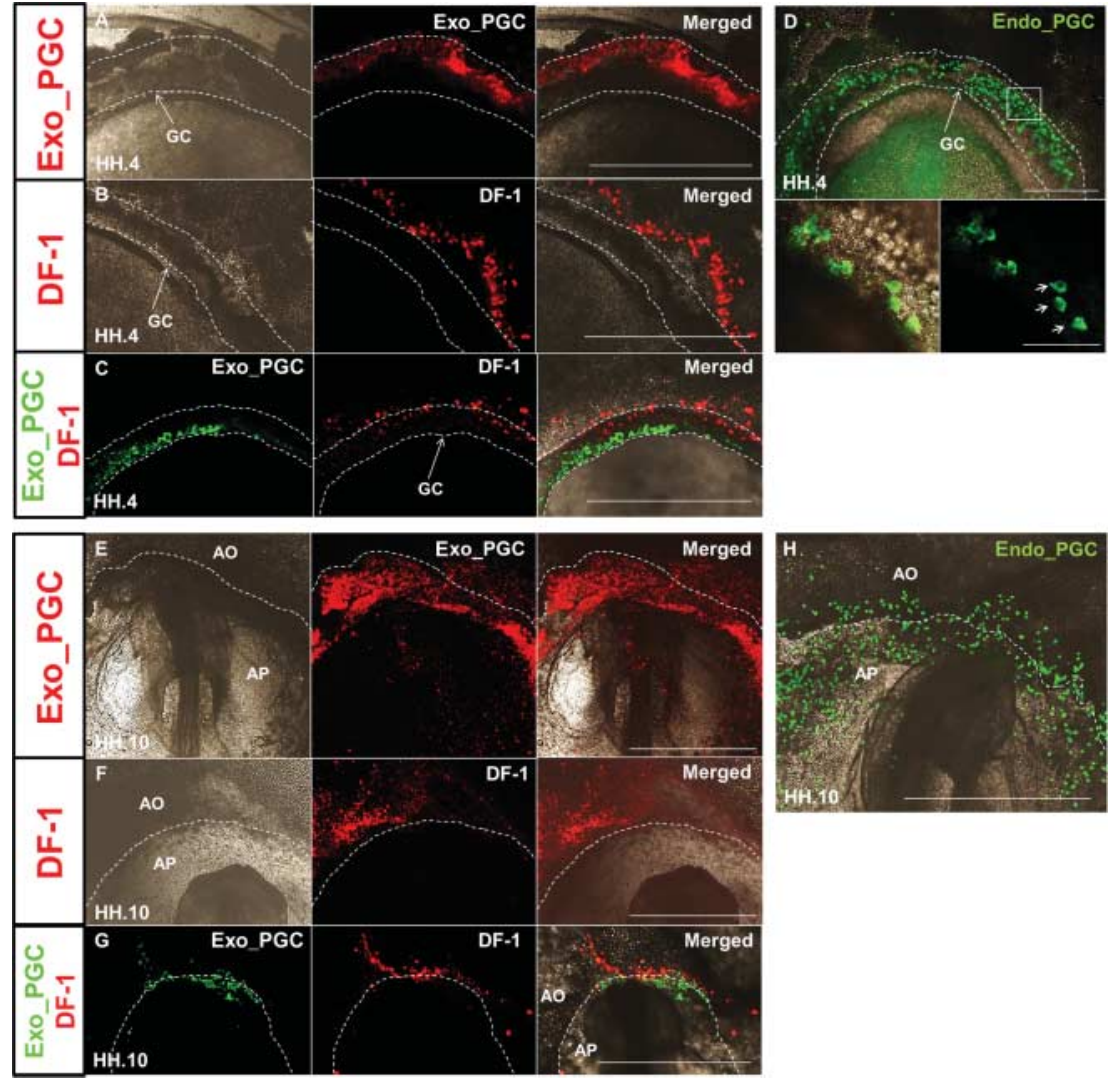

Figure 4 Differences in position between exogenous PGCs and DF- 1 cells at $\mathrm{HH}$ stage 4 and 10. After PKH26-labeled PGCs, PKH26labeled DF-1 cells, or both GFP_PGCs and PKH26-labeled DF-1 cells were transplanted into the subgerminal cavity in EGK stage $X$ embryos, transplanted and non-transplanted embryos were incubated until $\mathrm{HH}$ stage 4 (A, B, $\mathrm{C}$, and $\mathrm{D}$ ) and $\mathrm{HH}$ stage 10 (E, F, G, and $\mathrm{H})$. After incubation, the non-transplanted embryos were immunostained with anti-cDAZL antibody $(\mathrm{D}$ and $\mathrm{H}$ ) and observed under a microscope. At $\mathrm{HH}$ stage 4, PKH26-labeled or GFP-expressing exogenous PGCs and cDAZLexpressing endogenous PGCs were observed in the germinal crescent (GC, dotted lines) (A, C, and D), while most exogenous DF-1 cells were not detected in the $\mathrm{GC}$ region (B and $\mathrm{C}$ ). White arrows in the magnified view (D) show the polarized and amoeboid morphology of endogenous PGCs. At HH stage 10, PKH26labeled, or GFP-expressing exogenous PGCs and CDAZL-expressing endogenous PGCs were located in the anterior region of the area pellucida (AP) near the embryonic head (E, G, and $\mathrm{H}$ ), while exogenous DF- 1 cells were observed in the area opaca (AO) (F and G). The dotted lines in $(E, F, G$, and $H)$ indicate the margin of the area opaca. Scale bars $=1000 \mu \mathrm{m}$ (A, B, and C and E, F, G, and H), $500 \mu \mathrm{m}(\mathrm{D})$, and $100 \mu \mathrm{m}$ (magnified view of (D)). 


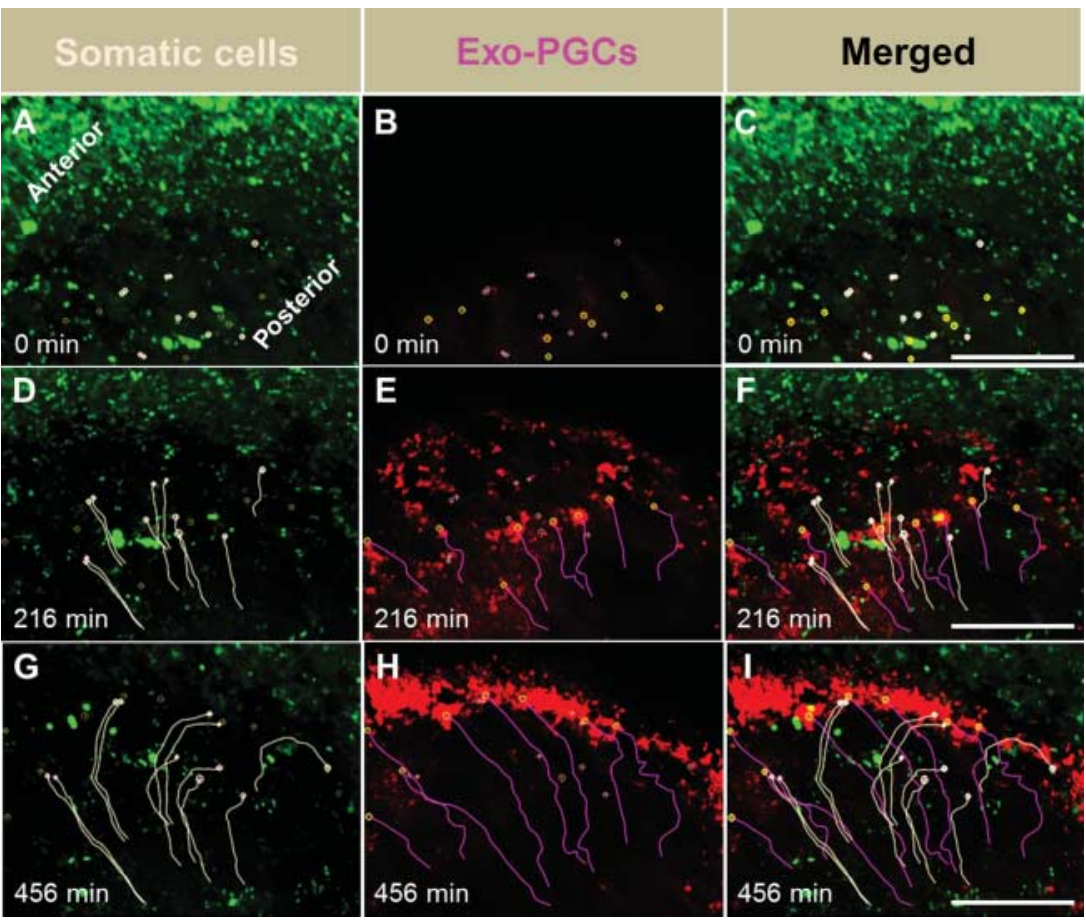

Figure 5 Migration pathway of PGCs between EGK stage $\mathrm{X}$ and $\mathrm{HH}$ stage 2. PKH26-labeled PGCs were transplanted into the subgerminal cavity in GFP-expressing TG embryos at EGK stage X. The embryos were then cultured in the live-imaging chamber for $8 \mathrm{~h}$ with time-lapse imaging using a confocal microscope. (A, D, and G) Tracking of somatic cells showing normal epiblast movement (white lines). (B, E, and $\mathrm{H}$ ) Tracking of exogenous PGCs showing migration toward the anterior region (yellow lines). (C, F, and I) Merged images of all channels. For all images, the anteroposterior axis is from the upper left side to the lower right side. Scale bars $=500 \mu \mathrm{m}$.

\section{Active migration of PGCs toward the germinal crescent region}

To clarify the ability of PGCs to migrate toward the germinal crescent region after injection into the subgerminal cavity, PKH26-labeled or GFP-expressing cell-transplanted embryos and non-transplanted embryos were incubated until $\mathrm{HH}$ stage 4 . At $\mathrm{HH}$ stage 4 , the non-transplanted embryos were immunostained with anti-cDAZL antibody to identify endogenous PGCs and to compare their location with that of exogenous PGCs. Most of the exogenous PGCs were located in the germinal crescent region (Fig. $4 \mathrm{~A}$ and $\mathrm{C}$ ), as were most cDAZL-expressing endogenous PGCs (Fig. 4D). In contrast, most DF-1 cells were located outside of the germinal crescent region (Fig. $4 \mathrm{~B}$ and $\mathrm{C}$ ). Thus, the exogenous PGCs and DF-1 cells were differently located in relation to the germinal crescent region, although both cell types migrated toward the anterior zone. In addition, cDAZL-expressing endogenous PGCs located laterally in the anterior region showed a polarized and amoeboid morphology, an indicator of active cell migration (Fig. 4D).

\section{Active migration of PGCs toward the anterior head region before they enter the blood circulation}

To investigate the ability of PGCs to migrate toward the anterior head region before they enter the circulation, PKH26-labeled or GFP-expressing cell-transplanted embryos and non-transplanted embryos were incubated until $\mathrm{HH}$ stage 10 . At $\mathrm{HH}$ stage 10, non-transplanted embryos were immunostained with anti-cDAZL antibody to identify endogenous PGCs and to compare their location with that of exogenous PGCs. Most of the exogenous PGCs were located in the anterior region of the area pellucida near the embryonic head (Fig. 4E and G). cDAZL-expressing endogenous PGCs were found in the same region (Fig. 4H). DF-1 cells were detected laterally in the area opaca, away from the anterior head (Fig. 4F and G).

\section{Tracking PGCs between EGK stage $X$ and $\mathrm{HH}$ stage 2 by time-lapse imaging}

We confirmed that subgerminal cavity-transplanted PGCs but not DF- 1 cells can actively migrate into the germinal crescent region at $\mathrm{HH}$ stage 4 and incorporate into the anterior region of the area pellucida, although both cell types passively migrate toward the anterior marginal region. To clarify the mechanism of PGC migration toward the germinal crescent region, time-lapse live imaging of the embryos for $8 \mathrm{~h}$ (EGK stage $\mathrm{X}$ to $\mathrm{HH}$ stage 2 ) was done after PKH26-labeled PGCs were transplanted into the subgerminal cavity of GFP-expressing TG embryos at EGK stage X (Fig. 5). The TG embryos express GFP ubiquitously under the control of the CMV promoter (Park \& Han 2012). Then, the movements of several cells located in the centers of embryos were monitored. Some of the endogenous cells in the central axis at EGK stage $X$ migrated in a straight line 

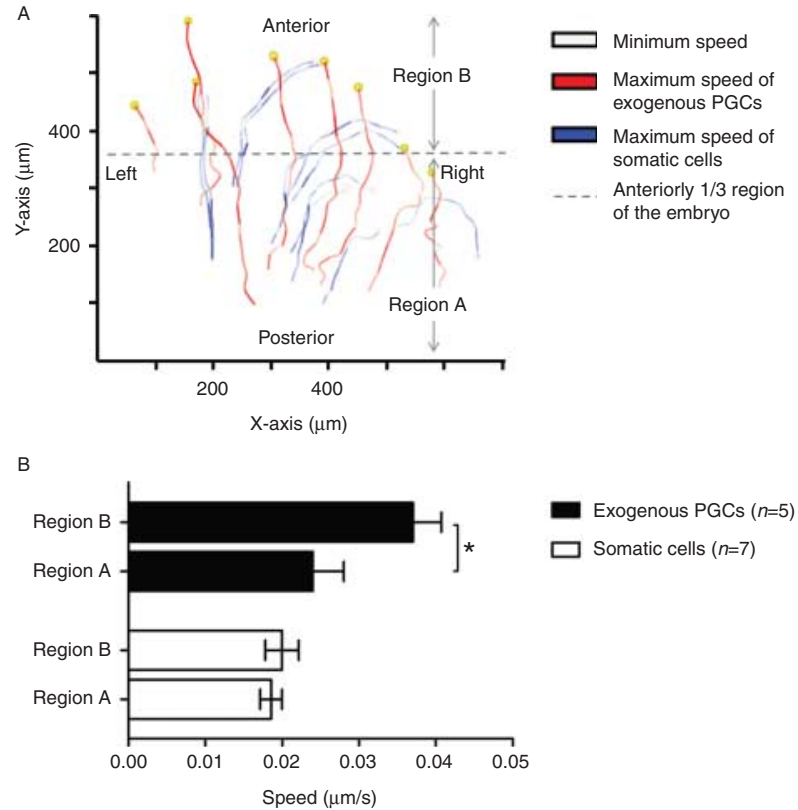

Figure 6 Space-specific increase in migratory speed in PGCs. Migratory tracks of PGCs compared with neighboring somatic cells were investigated between EGK stage $\mathrm{X}$ and $\mathrm{HH}$ stage 2 according to spatial and temporal criteria. (A) Line plots showing the migratory tracks of exogenous PGCs compared with neighboring somatic cells, indicating that PGCs increased their speed when they reached the anterior onethird of the embryo. (B) The mean speed of exogenous PGCs was significantly higher in region $B$ compared with region $A$, while the mean speed of somatic cells showed no significant change. The bars in (B) represent the SEM of five exogenous PGCs and seven somatic cells. ${ }^{*} P<0.05$, region $\mathrm{A}$ vs $\mathrm{B}$.

and reached the anterior region, while other endogenous cells located laterally migrated in a curved or circular line (Fig. 5A, D, and G). These movements are identical to the normal migration of epiblast cells (Voiculescu et al. 2007). However, the PKH26-labeled exogenous PGCs located differently in the central regions of embryos migrated toward the anterior region in a straight line and settled in the future germinal crescent region at $\mathrm{HH}$ stage 2 (Fig. 5B, $\mathrm{E}$, and $\mathrm{H}$ ). Thus, the exogenous PGCs showed apparent differences in migration pattern compared with somatic cells (Fig. 5C, F, and I).

Since most of the traced cells showed anterior movement initially, changes in the migration speed of exogenous PGCs and somatic cells were investigated to clarify when and where active and passive forces of migration act (Fig. 6). During the initial $4 \mathrm{~h}$ of culture (EGK stages X-XII), all selected cells maintained a constant speed until they reached the anterior onethird of the embryo. After $4 \mathrm{~h}$, exogenous PGCs exhibited faster migration while the somatic cells maintained their constant speed (Fig. 6A). The mean speed of exogenous PGCs increased significantly $(P<0.05)$ after they reached the anterior one-third of the embryo, while that of somatic cells showed no significant change (Fig. 6B). When PKH26-labeled DF-1 cells were transplanted, they maintained a constant speed until they reached the anterior one-third of the embryo, as somatic cells did (Supplementary Fig. S2, see section on supplementary data given at the end of this article). When we observed the morphological changes in the exogenous PGCs during their fast migration (Fig. 6), most of the cells exhibited successive contraction and expansion, indicating active migration (Fig. 7).

\section{Discussion}

The migration pathway of chicken PGCs before they settle in the embryonic gonads has been determined by studying the relative positions of PGCs during embryonic development. PGCs located in the central region of the area pellucida during cleavage stages populate the germinal crescent, the anterior arc-like border region between the area pellucida and area opaca, at $\mathrm{HH}$ stage 4 (Fig. 8A), and migrate toward the genital ridges via the circulation (Swift 1914, Fujimoto et al. 1976). However, details of the underlying mechanisms at each time point are largely unknown. In this study, we demonstrated the passive and active migration of PGCs, and the importance of PGCs' initial location in determination of cell migration toward the germinal crescent.

First, we confirmed the previous idea that PGCs migrate passively by morphogenetic movement of the hypoblast (Ginsburg \& Eyal-Giladi 1986). When transplanted into the subgerminal cavity in EGK stage $X$ embryos, PGCs and somatic (DF-1) cells were located in the anterior border region at $\mathrm{HH}$ stage 4 . These results indicate that when the cells land on the hypoblast, they are passively carried by the anterior movement of the hypoblast, as PGCs are if they are
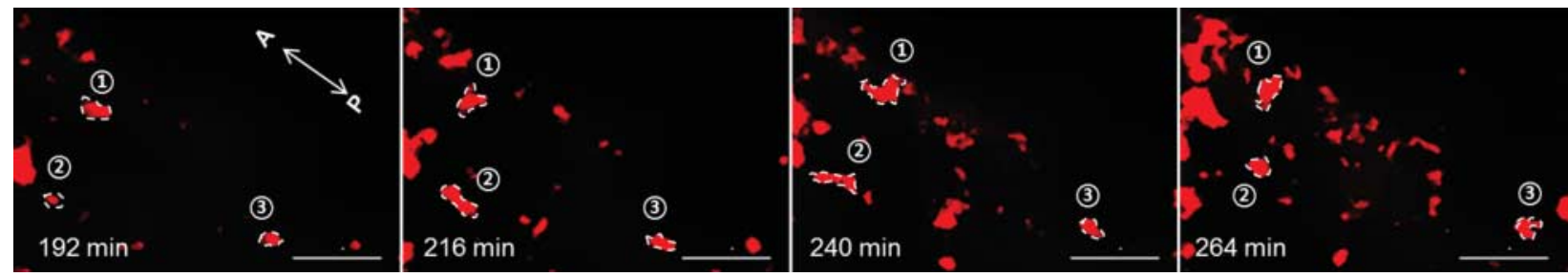

Figure 7 Amoeboid movement of chicken PGCs toward the germinal crescent. Morphological changes in PKH26-labeled exogenous PGCs in GFP-TG embryos were monitored between EGK stage X and HH stage 2. Magnified and sequential images of exogenous PGCs showing dynamic morphological changes and amoeboid movement toward the anterior region (dotted lines and circled number). For all images, the anteroposterior axis is from the upper left side to the lower right side. Scale bars $=100 \mu \mathrm{m}$. 


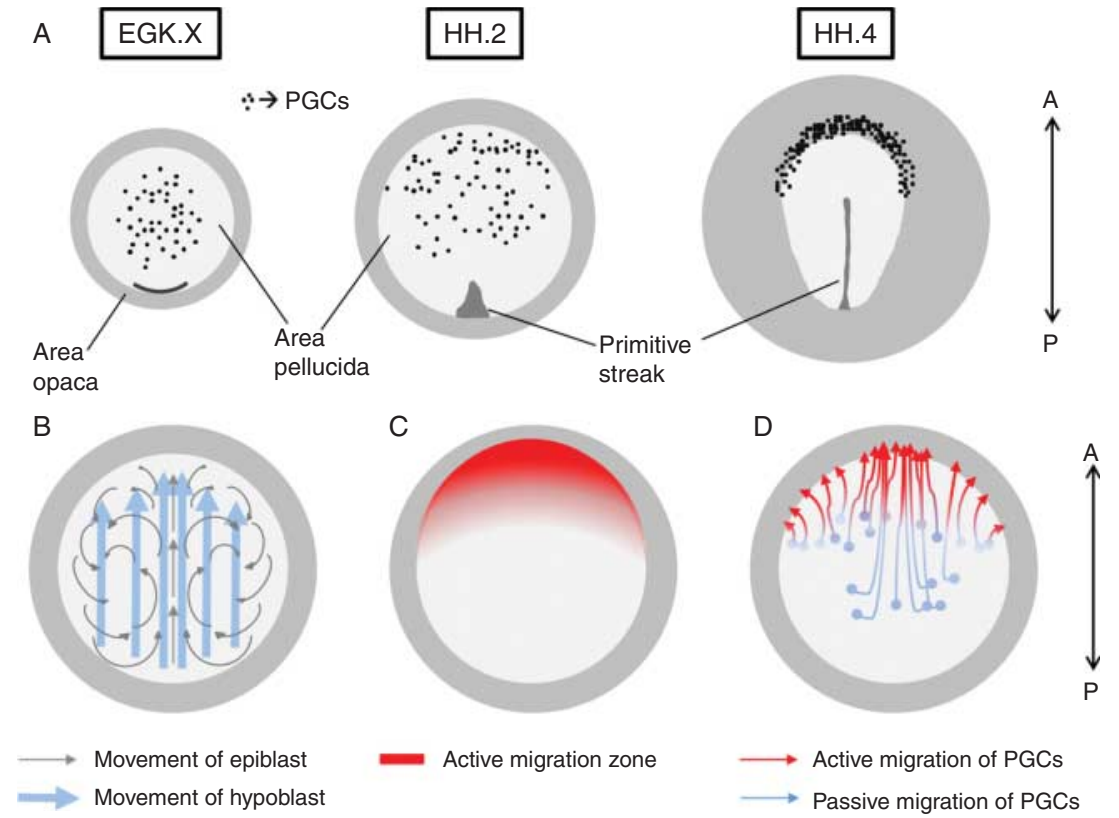

Figure 8 Proposed hypothesis for migration of chicken PGCs toward the germinal crescent. (A) Location of PGCs between EGK stage $X$ and $\mathrm{HH}$ stage 4 . At EGK stage $\mathrm{X}$, PGCs are located in the central region of the area pellucida. By $\mathrm{HH}$ stage 4, most of the PGCs have reached the germinal crescent region. (B) Global movement of embryonic cells, including epiblast and hypoblast cells, between EGK stage $\mathrm{X}$ and $\mathrm{HH}$ stage 2 . (C) Active migration zone between EGK stage $X$ and $\mathrm{HH}$ stage 2, covering the anterior part of the embryo. (D) Migration pathway of PGCs toward the germinal crescent. Once PGCs reach the anterior region passively by embryonic flow (blue lines), they start actively migrating toward the germinal crescent region (red lines). translocated from the epiblast to the hypoblast (Swift 1914, Eyal-Giladi et al. 1981, Ginsburg \& Eyal-Giladi 1986). Next, to further investigate the passive force toward the anterior region, $\mathrm{PKH} 26$-labeled exogenous cells were transplanted into the PMZ in EGK stage $X$ embryos. Notably, PGCs and DF-1 cells did not reach the anterior region when the embryos were incubated until $\mathrm{HH}$ stage 10, which indicates that for normal migration towards anterior region, the initial location of PGCs should be the central region of the embryo. In our study, PGCs transplanted into the PMZ did not migrate into the embryonic gonads. Because PGCs should be positioned in the anterior region before entry into the embryonic circulation to use the anterior vitelline veins (De Melo Bernardo et al. 2012), it seems reasonable that the site of PGC formation is the anterior region, not the central region as was thought. However, the anteroposterior (AP) axis does not develop at the time of PGC emergence in chicken. The cVG1 gene, an initiator of axis formation, is known to be expressed from EGK stage $X$ (Seleiro et al. 1996), and the first morphological indication of the AP axis formation of the area pellucida is in EGK stage VII (Eyal-Giladi \& Kochav 1976), although this is not well understood at the molecular level. However, chicken PGCs emerge at the initial cleavage stages (Tsunekawa et al. 2000).

We confirmed that PGCs migrate passively toward the anterior marginal region of the embryo during hypoblast formation and demonstrated the importance of the initial location of PGCs. In a previous study, however, PGCs near the germinal crescent around $\mathrm{HH}$ stage 4 showed an amoeboid morphology, indicating active cell migration (Ginsburg \& Eyal-Giladi 1986). Therefore, to confirm whether migration was only passive, we magnified the germinal crescent region with immunostaining of endogenous PGCs. Only exogenous PGCs were located in the germinal crescent, where endogenous PGCs are positive for cDAZL. DF-1 cells were clearly located away from the germinal crescent. Also, many endogenous PGCs showed an amoeboid morphology at $\mathrm{HH}$ stage 4 . These results indicate that although both PGCs and DF- 1 cells can migrate passively toward the anterior region, only PGCs can gather in the germinal crescent by active migration. When the embryos were further incubated to $\mathrm{HH}$ stage 10 after cell transplantation, only exogenous PGCs were located in the anterior region of the area pellucida, where endogenous PGCs are positive for CDAZL. PGCs located in this area are ready to enter the blood circulation through veins (Nakamura et al. 2007). However, most of the DF-1 cells were not located in this region. These results suggest that PGCs actively migrate toward the germinal crescent at $\mathrm{HH}$ stage 4 and the anterior region of the area pellucida at $\mathrm{HH}$ stage 10 . To further clarify this point, molecular mechanisms governed by signaling should be investigated in further studies.

Based on our results, both passive and active forces are crucial for PGC migration. We further examined spatial and temporal aspects of passive and active migration through cell-tracking experiments with live imaging using GFP-TG. Centrally located exogenous PGCs at EGK stage $X$ showed rapid movement toward the anterior border region, including both sides of the anterolateral regions, and most of them had already arrived at $\mathrm{HH}$ stage 2 . When we checked the relative positions of endogenous PGCs during embryonic development by wholemount in situ hybridization, many of them were still located in the central region 
of the embryo at $\mathrm{HH}$ stage 2 (others were in the germinal crescent). This discrepancy between exogenous and endogenous PGCs can be explained by the difference in their initial positions (Ginsburg \& Eyal-Giladi 1986). Unlike the exogenous PGCs, which are located in the hypoblast under the epiblast after injection into the subgerminal cavity, endogenous PGCs migrate gradually from the epiblast toward the hypoblast until $\mathrm{HH}$ stage 4 (Ginsburg \& Eyal-Giladi 1986). During their movement, PGCs exhibited morphological changes, with successive contraction and expansion, indicating active migration (Ridley et al. 2003). Endogenous somatic cells expressing GFP maintained normal epiblast movement (Hatada \& Stern 1994). Also, the migration speed of the exogenous PGCs increased markedly after $4 \mathrm{~h}$ of incubation when the PGCs reached the anterior onethird of the embryo. In contrast, somatic cells and DF-1 cells maintained a constant speed throughout the observation period. The dynamic increase in migration speed might be due to a gradient of chemoattractant spreading from the germinal crescent to the anterior one-third of the embryo (Bianco et al. 2007, Mizoguchi et al. 2008).

In this report, we propose a hypothesis for PGC migration toward the germinal crescent. After specification, PGCs are located in the central epiblast or hypoblast until EGK stage $X$. Then, they migrate toward the anterior region passively by morphogenetic radial movement of epiblast or linear movement of the hypoblast in the posteroanterior direction (Fig. 8B). When the PGCs reach the anterior one-third of the embryo, they migrate actively toward the germinal crescent with increased speed, possibly in response to an unknown chemoattractant or chemorepellent (Fig. 8C). We conclude that chicken PGCs use sequential passive and active forces to migrate toward the germinal crescent (Fig. 8D).

\section{Supplementary data}

This is linked to the online version of the paper at http://dx.doi. org/10.1530/REP-14-0433.

\section{Declaration of interest}

The authors declare that there is no conflict of interest that could be perceived as prejudicing the impartiality of the research reported.

\section{Funding}

This work was supported by a grant from the Next-Generation BioGreen 21 Program (PJ0081422014) and from the Cooperative Research Program for Agriculture Science and Technology Development (project no. PJ010390012014) of the Rural Development Administration, Republic of Korea.

\section{References}

Bianco A, Poukkula M, Cliffe A, Mathieu J, Luque CM, Fulga TA \& Rorth P 2007 Two distinct modes of guidance signalling during collective migration of border cells. Nature 448 362-365. (doi:10.1038/ nature05965)

De Melo Bernardo A, Sprenkels K, Rodrigues G, Noce T \& Chuva De Sousa Lopes SM 2012 Chicken primordial germ cells use the anterior vitelline veins to enter the embryonic circulation. Biology Open 1 1146-1152. (doi:10.1242/bio.20122592)

Extavour CG \& Akam M 2003 Mechanisms of germ cell specification across the metazoans: epigenesis and preformation. Development 130 5869-5884. (doi:10.1242/dev.00804)

Eyal-Giladi H \& Kochav S 1976 From cleavage to primitive streak formation: a complementary normal table and a new look at the first stages of the development of the chick. I. General morphology. Developmental Biology 49 321-337. (doi:10.1016/0012-1606(76) 90178-0)

Eyal-Giladi H, Ginsburg M \& Farbarov A 1981 Avian primordial germ cells are of epiblastic origin. Journal of Embryology and Experimental Morphology 65 139-147.

Franz CM, Jones GE \& Ridley AJ 2002 Cell migration in development and disease. Developmental Cell 2 153-158. (doi:10.1016/S1534-5807 (02)00120-X)

Fujimoto T, Ukeshima A \& Kiyofuji R 1976 The origin, migration and morphology of the primordial germ cells in the chick embryo. Anatomical Record 185 139-145. (doi:10.1002/ar.1091850203)

Ginsburg M \& Eyal-Giladi H 1986 Temporal and spatial aspects of the gradual migration of primordial germ cells from the epiblast into the germinal crescent in the avian embryo. Journal of Embryology and Experimental Morphology 95 53-71.

Ginsburg M \& Eyal-Giladi H 1987 Primordial germ cells of the young chick blastoderm originate from the central zone of the area pellucida irrespective of the embryo-forming process. Development 101 209-219.

Hamburger V \& Hamilton HL 1951 A series of normal stages in the development of the chick embryo. Journal of Morphology 88 49-92. (doi:10.1002/jmor.1050880104)

Hamburger V \& Hamilton HL 1992 A series of normal stages in the development of the chick embryo. 1951. Developmental Dynamics 195 231-272. (doi:10.1002/aja.1001950404)

Hatada Y \& Stern CD 1994 A fate map of the epiblast of the early chick embryo. Development 120 2879-2889.

Lauffenburger DA \& Horwitz AF 1996 Cell migration: a physically integrated molecular process. Cell 84 359-369. (doi:10.1016/S00928674(00)81280-5)

Mizoguchi T, Verkade H, Heath JK, Kuroiwa A \& Kikuchi Y 2008 Sdf1/Cxcr4 signaling controls the dorsal migration of endodermal cells during zebrafish gastrulation. Development 135 2521-2529. (doi:10. 1242/dev.020107)

Nakamura Y, Yamamoto Y, Usui F, Mushika T, Ono T, Setioko AR, Takeda K, Nirasawa K, Kagami H \& Tagami T 2007 Migration and proliferation of primordial germ cells in the early chicken embryo. Poultry Science $\mathbf{8 6}$ 2182-2193. (doi:10.1093/ps/86.10.2182)

Park TS \& Han JY 2012 piggyBac transposition into primordial germ cells is an efficient tool for transgenesis in chickens. PNAS 109 9337-9341. (doi:10.1073/pnas.1203823109)

Rengaraj D, Zheng YH, Kang KS, Park KJ, Lee BR, Lee SI, Choi JW \& Han JY 2010 Conserved expression pattern of chicken DAZL in primordial germ cells and germ-line cells. Theriogenology 74 765-776. (doi:10.1016/j. theriogenology.2010.04.001)

Richardson BE \& Lehmann R 2010 Mechanisms guiding primordial germ cell migration: strategies from different organisms. Nature Reviews. Molecular Cell Biology 11 37-49. (doi:10.1038/nrm2815)

Ridley AJ, Schwartz MA, Burridge K, Firtel RA, Ginsberg MH, Borisy G, Parsons JT \& Horwitz AR 2003 Cell migration: integrating signals from front to back. Science 302 1704-1709. (doi:10.1126/science. 1092053)

Seleiro EA, Connolly DJ \& Cooke J 1996 Early developmental expression and experimental axis determination by the chicken Vg1 gene. Current Biology 6 1476-1486. (doi:10.1016/S0960-9822(96)00752-X) 
Stebler J, Spieler D, Slanchev K, Molyneaux KA, Richter U, Cojocaru V, Tarabykin V, Wylie C, Kessel M \& Raz E 2004 Primordial germ cell migration in the chick and mouse embryo: the role of the chemokine SDF-1/CXCL12. Developmental Biology 272 351-361. (doi:10.1016/j. ydbio.2004.05.009)

Stern CD 1998 Detection of multiple gene products simultaneously by in situ hybridization and immunohistochemistry in whole mounts of avian embryos. Current Topics in Developmental Biology 36 223-243.

Swift CH 1914 Origin and early history of the primordial germ-cells in the chick. American Journal of Anatomy 15 483-515. (doi:10.1002/aja. 1000150404)

Tsunekawa N, Naito M, Sakai Y, Nishida T \& Noce T 2000 Isolation of chicken vasa homolog gene and tracing the origin of primordial germ cells. Development 127 2741-2750.
Voiculescu O, Bertocchini F, Wolpert L, Keller RE \& Stern CD 2007 The amniote primitive streak is defined by epithelial cell intercalation before gastrulation. Nature 449 1049-1052. (doi:10.1038/ nature06211)

Yalcin HC, Shekhar A, Rane AA \& Butcher JT 2010 An ex-ovo chicken embryo culture system suitable for imaging and microsurgery applications. Journal of Visualized Experiments 44 2154. (doi:10.3791/2154)

Received 20 August 2014

First decision 29 September 2014

Revised manuscript received 8 November 2014

Accepted 2 December 2014 